\title{
Molecular association of glucose-6- phosphate isomerase and pyruvate kinase M2 with glyceraldehyde-3-phosphate dehydrogenase in cancer cells
}

Mahua R. Das ${ }^{1+}$, Arup K. Bag ${ }^{2 \dagger}$, Shekhar Saha ${ }^{1}$, Alok Ghosh ${ }^{3}$, Sumit K. Dey ${ }^{1}$, Provas Das ${ }^{1}$, Chitra Mandal ${ }^{2}$, Subhankar Ray ${ }^{4 \wedge}$, Saikat Chakrabarti ${ }^{5}$, Manju Ray ${ }^{6^{*}}$ and Siddhartha S. Jana ${ }^{1^{*}}$

\begin{abstract}
Background: For a long time cancer cells are known for increased uptake of glucose and its metabolization through glycolysis. Glyceraldehyde-3-phosphate dehydrogenase (GAPDH) is a key regulatory enzyme of this pathway and can produce ATP through oxidative level of phosphorylation. Previously, we reported that GAPDH purified from a variety of malignant tissues, but not from normal tissues, was strongly inactivated by a normal metabolite, methylglyoxal (MG). Molecular mechanism behind MG mediated GAPDH inhibition in cancer cells is not well understood.

Methods: GAPDH was purified from Ehrlich ascites carcinoma (EAC) cells based on its enzymatic activity. GAPDH associated proteins in EAC cells and 3-methylcholanthrene (3MC) induced mouse tumor tissue were detected by mass spectrometry analysis and immunoprecipitation (IP) experiment, respectively. Interacting domains of GAPDH and its associated proteins were assessed by in silico molecular docking analysis. Mechanism of MG mediated GAPDH inactivation in cancer cells was evaluated by measuring enzyme activity, Circular dichroism (CD) spectroscopy, IP and mass spectrometry analyses.

Result: Here, we report that GAPDH is associated with glucose-6-phosphate isomerase (GPI) and pyruvate kinase M2 (PKM2) in Ehrlich ascites carcinoma (EAC) cells and also in 3-methylcholanthrene (3MC) induced mouse tumor tissue. Molecular docking analyses suggest C-terminal domain preference for the interaction between GAPDH and GPI. However, both $C$ and $N$ termini of PKM2 might be interacting with the $C$ terminal domain of GAPDH. Expression of both PKM2 and GPI is increased in 3MC induced tumor compared with the normal tissue. In presence of $1 \mathrm{mM} \mathrm{MG}$, association of GAPDH with PKM2 or GPI is not perturbed, but the enzymatic activity of GAPDH is reduced to $26.8 \pm 5 \%$ in $3 \mathrm{MC}$ induced tumor and $57.8 \pm 2.3 \%$ in EAC cells. Treatment of MG to purified GAPDH complex leads to glycation at $\mathrm{R} 399$ residue of PKM2 only, and changes the secondary structure of the protein complex.

Conclusion: PKM2 may regulate the enzymatic activity of GAPDH. Increased enzymatic activity of GAPDH in tumor cells may be attributed to its association with PKM2 and GPI. Association of GAPDH with PKM2 and GPI could be a signature for cancer cells. Glycation at R399 of PKM2 and changes in the secondary structure of GAPDH complex could be one of the mechanisms by which GAPDH activity is inhibited in tumor cells by MG.
\end{abstract}

Keywords: Glyceraldehyde-3-phosphate dehydrogenase, Glucose-6-phosphate isomerase, Pyruvate kinase M2, Molecular association, Malignancy

\footnotetext{
*Correspondence: manjuray@mail.jcbose.ac.in; bcssj@iacs.res.in

${ }^{\dagger}$ Equal contributors

Deceased

${ }^{6}$ Department of Biophysics, Bose Institute, Kolkata, India

'Department of Biological Chemistry, Indian Association for the Cultivation of

Science, Kolkata 700032, India

Full list of author information is available at the end of the article
} 


\section{Background}

An important characteristic of rapidly proliferating malignant cells is their capacity of high aerobic glycolysis. Cancer cells display a high level of glucose uptake and enhanced lactic acid production as compared to normal cells, a phenomenon widely known as the Warburg effect [1]. Glyceraldehyde-3-phosphate dehydrogenase (GAPDH, EC 1.2.1.12) is a highly conserved and important enzyme for catalyzing the 6th step of glycolysis by conversion of glyceraldehyde-3-phosphate (GAP) and inorganic phosphate (Pi) into 1, 3-bisphosphoglycerate (1, 3-BPG) in the presence of $\mathrm{NAD}^{+}$. GAPDH has been shown to exhibit a wide variety of cellular functions like apoptosis, neurotransmission, phagocytosis, and vesicle fusion in ER to Golgi transport etc., besides its role as a glycolytic enzyme [2-6]. Interestingly, GAPDH can also translocate to the nucleus. Induction of GAPDH translocation to nucleus causes cell death in human SH-SY5Y neuroblastoma and rat neonatal cardiomyocytes $[7,8]$ and lowering such nuclear localization suppresses apoptosis in ovarian cancer cells [9]. Recent studies have established enhanced expression of both mRNA and protein level of GAPDH in human lung cancer tissues, pancreatic adenocarcinomas, and dunning rat prostate cancer cell lines compared with normal tissues and cell lines [10-12]. High expression of GAPDH has been correlated with upregulation of cell cycle related genes that are involved in G2/M transition, $M$ phase regulation, glycolytic genes, and down regulation of gluconeogenesis in non-small cell lung carcinoma [13].

GAPDH is a homotetrameric protein with molecular mass of $145 \mathrm{kDa}$. Each identical subunit contains 333 residues with molecular mass of $35.9 \mathrm{kDa}$ [14]. In contrast, Bagui et al. [15] and Patra et al. [16] showed that GAPDH is a heterodimer consisting of two non identical subunits approximately of 33 and $55 \mathrm{kDa}$ when purified from Ehrlich ascites carcinoma (EAC) cells, mouse sarcoma tissue, and human leukemic leukocytes. Interestingly, methylglyoxal (MG), a normal metabolite could inactivate GAPDH activity in a wide variety of malignant cells and tissues, whereas it has no effect on GAPDH from normal sources [17]. However, the mechanism behind MG mediated inhibition of GAPDH activity in cancer cells is unknown.

Here, we report for the first time that purified GAPDH complex purified from EAC cells contains two distinct $55 \mathrm{kDa}$ subunits associated with a $33 \mathrm{kDa}$ subunit. We identify these two $55 \mathrm{kDa}$ subunits as two glycolytic enzymes- one is GPI and another is PKM2. GAPDH can interact with both of these enzymes in EAC cells as well as in $3 \mathrm{MC}$ induced tumor tissue. We establish that MG glycates R399 residue located in M2 insert of PKM2 and changes the secondary structure of the GAPDH complex. This study indicates a different molecular association of GAPDH and underscores the glycation events by methylglyoxal in cancer cells.

\section{Methods \\ Propagation of EAC cells}

EAC cells were maintained and grown in the peritoneal cavity of Swiss albino mice as described previously [18] following the guidelines of the Institutional Animal Ethics Committee of Indian Association for the Cultivation of Science (IACS) and ARRIVE guideline for reporting animal research. Guidelines were approved by the Institutional Animal Ethics Committee of IACS. Approximately, $10^{6}$ cells were diluted in $0.2 \mathrm{ml}$ of sterile normal saline $(0.9 \%)$ and were used for inoculums. The cells were harvested after 10-15 days. Erythrocytes were removed by washing with $0.45 \% \mathrm{NaCl}$.

\section{Mass spectrometry}

GAPDH was purified from EAC cells based on its enzymatic activity which was checked after each step of purification as described previously [15]. Purified GAPDH complex from EAC cells was run on 7.5-15\% SDS-PAGE, and bands were visualized by Coomassie blue stainining. Bands were excised from the gel and processed for mass spectrometry analysis separately. Briefly, after reduction, alkylation, and digestion with In-Gel tryptic Digestion Kit (Thermo Fisher Scientific, Waltham, MA, USA), the resulting peptide mixture was spotted using $\alpha$-cyano-4hydroxy cinnamic acid $(5.0 \mathrm{mg} / \mathrm{mL}$, Sigma) in $70 \%$ acetonitrile in $0.1 \%$ TFA and analyzed by MALDITOF/TOF (Applied Biosystem 4700, USA) mass spectrometer in reflector mode for protein identification by peptide mass fingerprint [19]. The mass spectrum was acquired using 4000 series explorer v3.5 software and peak list was generated between 800 and $4000 \mathrm{Da}$ with a signal-to-noise ratio of 25 . The searching parameters used for identification of acquired peptide spectrum were taxonomy: mouse; cleavage enzyme: trypsin; maximum number of miss cleavage 1; mass tolerance $100 \mathrm{ppm}$; peptide charge: +1 ; variable modification: oxidation of methionine and carbamidomethylation of cysteine, partial $\mathrm{N}$-terminal acetylation, modification of glutamine as fixed modification of peptides. Data was analysed using GPS explore v3.0 (TM) software. The combined MS and MS/MS results were matched with the NCBInr, SWISS PORT and MSDB databases using MASCOT software v2.1 in order to identify the proteins. Proteins were identified on the basis of significant MASCOT Mowse-score $(p<0.05$ where $\mathrm{p}$ is the probability that the observed match in a random event).

The quality and accuracy of the data were examined by determination of false discovery rate (FDR) values using Mascot software (Matrix Science, London, UK) as previously reported [20]. Briefly, Mascot generic format 
(MGF) file of raw combined MALDI-TOF and MALDITOF/TOF data were created using following parameters (MS peak filter: Peak density filter: 65/200 Da; S/N: 10; No of peaks: 200; Area: 50; MS/MS peak filter: Peak density filter: 65/200 Da; S/N: 5; No of peaks: 200; Area: 20). MGF files were submitted to Mascot website to calculate the FDR values of respective proteins. To find out the stable glycation product, we treated purified protein complex with $1 \mathrm{mM}$ MG for five days at room temperature [21]. Samples were run on 7.5-15 \% SDS-PAGE followed by mass spectroscopy analysis.

\section{Development of tumor in the hind leg of mice}

All animal experiments were carried out after receiving the approval of guidelines from the Institutional Animal Ethics Committee. Guidelines were adhered to ARRIVE for reporting animal research. Guidelines were approved by the Institutional Animal Ethics Committee. Mouse tumor was generated using a previously reported method [22]. Chemical carcinogen, 3MC, (Sigma-Aldrich, St. Louis, Missouri, USA) was injected intramuscularly into the left hind leg of Swiss albino female mice for three times with one week intervals. Each dose was $10 \mathrm{mg} \cdot \mathrm{kg}^{-1}$ bodyweight in $0.05 \mathrm{ml}$ of olive oil. At $98-105$ days, full grown tumor was developed at the site of $3 \mathrm{MC}$ injection in left leg whereas no tumor formation was visible in contra lateral right leg.

\section{Electrophoresis, immunoblot analysis and immunoprecipitation}

Mouse tissue and EAC cell extracts were prepared in modified radio immunoprecipitation assay buffer (RIPA, composed of $50 \mathrm{mM}$ Tris/ $\mathrm{HCl}$ of $\mathrm{pH}$ 8.0, $150 \mathrm{mM}$ sodium chloride, $1.0 \%$ Nonidet P-40, $4 \mathrm{mM}$ EDTA of $\mathrm{pH}$ 8.0) with $1 \mathrm{mM}$ dithiothreitol, $0.5 \mathrm{mM}$ phenylmethylsulfonyl fluoride and $1 \%$ protease inhibitor cocktails (Sigma-Aldrich) at $4 .{ }^{\circ} \mathrm{C}$ as previously reported $[22,23]$. Briefly, mouse tissue and EAC cell were homogenized in RIPA buffer and centrifuged at $10,000 \mathrm{~g}$ for $10 \mathrm{~min}$. The supernatant was fractionated by $8 \%$ SDS-PAGE and then transferred to polyvinylidene difluoride membranes. The membranes were blocked in PBS containing $5 \%$ BSA and $0.05 \%$ Tween-20 for $1 \mathrm{~h}$, and incubated overnight at $4^{0} \mathrm{C}$ with primary antibodies to GAPDH (1:4 000), GPI (1:4 000, Santa Cruz Biotechnology Inc., CA, USA), PKM2(1:3 000; Cell Signaling Technology, Danvers, MA, USA) or $\beta$ tubulin (1:4 000; Sigma-Aldrich). The membranes were then washed and incubated with horseradish peroxidaseconjugated secondary antibodies against mouse or rabbit IgG at room temperature for $2 \mathrm{~h}$ and developed with the supersignal west femto reagent (Thermo Fisher Scientific). Chemiluminescence signal was captured on Kodak film. Relative band intensity was quantified by using ImageJ software (NIH, Bethesda, MD, USA) after normalizing band intensity with $\beta$-tubulin.

For immunoprecipitation, tissue supernatants were incubated with specific primary antibodies for overnight at $4{ }^{\circ} \mathrm{C}$. Primary antibodies were then pooled down by incubating with protein $\mathrm{G}$ agarose for an additional $4 \mathrm{~h}$ at $4{ }^{\circ} \mathrm{C}$. Immunocomplexes were then subjected to western blot analysis as described previously. To detect GPI and PKM2 in the immunoprecipitate by western blot analysis, we used Veriblot IP (Abcam, Cambridge, UK) secondary antibody which detects only primary antibody used for western blot, but not for immunoprecipitation.

\section{Enzymatic assay}

Both EAC and mouse tissue extracts in RIPA buffer were used for enzymatic assay. GAPDH activity was assayed in Triethanolamine- $\mathrm{HCl}$ buffer, $\mathrm{pH} 8.5$ as described previously [15]. Briefly, $1 \mathrm{ml}$ of assay mixture contained $50 \mathrm{mM}$ triethanolamine buffer, $50 \mathrm{mM} \mathrm{Na}_{2} \mathrm{HPO}_{4}$ (Sigma-Aldrich), $0.2 \mathrm{mM}$ EDTA, $0.5 \mathrm{mM} \mathrm{NAD}^{+}$and $0.04 \mathrm{mM}$ of Dglyceraldehyde- 3-phosphate (Sigma-Aldrich). The reaction was carried out by the addition of $5 \mu \mathrm{g}$ extracts, and monitored by recording absorbance of NADH at $340 \mathrm{~nm}$ at $30 \mathrm{~s}$ intervals. Increase in the absorbance values remained almost linear for $3 \mathrm{~min}\left(\triangle \mathrm{A}: 0.025-0.040 \mathrm{~min}^{-1}\right)$. One unit of activity of GAPDH was defined as the amount of enzyme required to convert $1 \mu \mathrm{mol}$ of $\mathrm{NAD}^{+}$to $\mathrm{NADH}$ per min under standard assay conditions. The specific activity was calculated as units of activity present per mg of protein. For MG mediated inhibition study, $30 \mu \mathrm{g}$ of protein from $3 \mathrm{MC}$ induced tumor tissue and EAC cell lysates were preincubated with $0-1 \mathrm{mM} \mathrm{MG}$ for $10 \mathrm{~min}$ at $25{ }^{\circ} \mathrm{C}$, and enzymatic activity of GAPDH and GPI was measured. We followed resorcinol method developed by Roe et al. [24] to measure GPI activity. Briefly, resorcinol detects the keto group present in fructose-6-phosphate. Samples from 3MC induced tumors and EAC were incubated in $50 \mathrm{mM}$ Tris / $\mathrm{HCl}$ buffer of $\mathrm{pH} 7.4$ with $1 \mathrm{mM}$ of substrate glucose-6phosphate in presence and absence of MG at different concentration for $10 \mathrm{~min} .1 \mathrm{~g} / 100 \mathrm{ml}$ resorcinol solution and $10 \mathrm{~N} \mathrm{HCl}$ at 1:7 ratio were added to the reaction and kept for $10 \mathrm{~min}$ at $80{ }^{\circ} \mathrm{C}$. Absorbance at $520 \mathrm{~nm}$ of was recorded using Varioskan Flash Elisa Reader (Thermo Fisher Scientific). Absorbance of MG alone was subtracted from that of resorcinol-fructose condensation product. Percent of GAPDH or GPI activity was calculated by considering specific activity of untreated sample as 100 .

\section{Molecular in silico docking analysis}

Three-dimensional (3D) structures of human GAPDH (Protein Data Bank (PDB) code: 1U8F, chain O) [25], PKM2 (PDB code: 1ZJH, chain A) and GPI (PDB code: 1JLH, chain A) [26] were collected from the PDB database [27]. 3D co-ordinates of GAPDH - PKM2 and 
GAPDH - GPI were further utilized for blind docking approach using three different protein-protein docking programs, ClusPro [28, 29], PatchDock [30] and SwarmDock [31], respectively. Top 30 docking solutions from each of the program's output were selected and were further utilized to identify the interacting interface using the PDBePISA server [32]. Residues involved in the docking interface from each protein chain were identified using inhouse perl scripts. Domain involvement of a protein for a docking complex is assigned if $60 \%$ or more residues from the $\mathrm{N}$ or $\mathrm{C}$ terminal domains are involved in forming the interface. Frequency of hydrogen bonding, salt bridge formation and change in free energy $(\Delta G)$ of interaction were extracted from the interface features calculated by the PDBePISA [32] program.

\section{Results}

PKM2 and GPI are associated with GAPDH in cancer cells Previous report by Bagui et al. [15] suggested that, on purification and molecular mass determination of GAPDH of EAC cells, $55 \mathrm{kDa}$ subunit is associated with $33 \mathrm{kDa}$ subunit of GAPDH. To characterize this unknown subunit, we purified GAPDH from EAC cells, based on its enzymatic activity (Additional file 1: Figure S1A-B). Additional file 1: Figure S1B shows that specific activity of GAPDH reached approximately 475 units/mg of protein, after passing through DEAE-Sephacell column. Interestingly, when the Sephacell column eluents were subjected to gradient SDS-PAGE, three bands of different sizes of approximately 58, 55 and $33 \mathrm{kDa}$ were visible with different intensities (Fig. 1a). We quantified the band intensity using ImageJ analysis and found that three polypeptides were co-purified at a $2.44: 4.35: 17.21$ molar ratio, respectively (Fig. 1b), suggesting that two types of polypeptides are associated with GAPDH at different amount in the purified protein complex of EAC cells.

To characterize the subunits associated with GAPDH during purification, we carried out MALDI-TOF/TOF experiments followed by peptide mass fingerprint (PMF) analysis of the three bands present in SDS-PAGE. Figure 1c shows the significantly identified proteins- PKM2 (from band 1), GPI (from band 2), and GAPDH (from band 3). We also performed false discovery rate (FDR) analysis to examine the quality and accuracy of the identified proteins. FDR values indicate that three proteins were identified with zero false positive identification. Sequences of trypsin digested fragments of the three individual bands were mapped with the NCBI database sequences of mouse PKM2 (KPYM_MOUSE), GPI (G6PI_MOUSE), and GAPDH (DEMSG). We found that sequence coverage for PKM2, GPI, and GAPDH were $21 \%, 45 \%$, and $24 \%$ respectively (Additional file 1: Figure S1C-E). A list of proteins detected from individual band in denatured condition is enlisted in Additional file 1: Table S1-3.
Detection of PKM2 and GPI by MALDI-TOF/TOF analysis prompted us to confirm these three proteins by immunoblot analysis. Figure 1d shows the detection of three glycolytic enzymes in the DEAE-Sephacell column eluents using specific antibody against each of these enzymes. Note that the amount of each enzyme increases in the eluents (lanes 3 and 4) compared with the crude lysates (lanes 1 and 2). Taken together, these results suggest that GAPDH isolated from EAC cells is associated with two glycolytic enzymes, PKM2 and GPI.

\section{Expression of PKM2 and GPI increases in tumor tissue}

To address the question whether this interaction of GAPDH with PKM2 and GPI occurs in other type of cancer cells, we used an in vivo tumor model system, in which tumor was developed in mice muscle by injecting a carcinogen, 3MC. We, first, checked the expression profile of these three glycolytic enzymes in 3MC induced tumor tissue by immunoblot analysis, and compared with that of the normal tissue from the contra lateral leg of same mouse. We found that both PKM2 and GPI were increased whereas GAPDH was reduced in the tumor (lane 3 and 4) compared with the normal tissue (lane 1 and 2, Fig. 2a). We quantified the expression of each of the enzyme and Fig. 2b shows that expression of GPI is higher by $2.2 \pm 0.45$ fold whereas as that GAPDH is lower by $1.8 \pm 0.22$ in $3 \mathrm{MC}$ induced tumor compared with normal tissue. On the other hand, PKM2 was not detectable in normal tissue. Christofk et al. [25] have recently shown that PKM2, but not PKM1 (another alternative spliced isoform of PKM), is advantageous for tumor cell growth and critical for tumorigenesis. We checked the expression of PKM1 in 3MC induced tumor tissue. Additional file 1: Figure S2 shows that PKM1 is detectable only in normal tissue but not in the $3 \mathrm{MC}$ induced tumor tissue, suggesting that tumor tissue expresses only PKM2.

Association of GAPDH with PKM2 and GPI in tumor cell was validated by immunoprecipitation assay. We immunoprecipitated GAPDH in normal and $3 \mathrm{MC}$ induced tumor tissue lysates using antibody against GAPDH, and the precipitate was further probed with antibodies against PKM2, GPI, and GAPDH. In Fig. 3a (and Additional file 1: Figure S3A-B), panels 1 and 2 show that both PKM2 and GPI are detectable in the immunoprecipitate of GAPDH antibody, but not of mouse IgG, in 3MC induced tumor tissue (lane 2). Panel 3 (Fig. 3a) indicates that GAPDH is immunoprecipitated specifically by GAPDH antibody, but not by mouse IgG, suggesting that both PKM2 and GPI are associated with GAPDH in tumor but not in normal tissue.

Also, we immunoprecipitated PKM2 in 3MC induced tumor tissue lysate, and probed with antibodies against GAPDH and GPI. Figure 3b shows that only GAPDH 
A

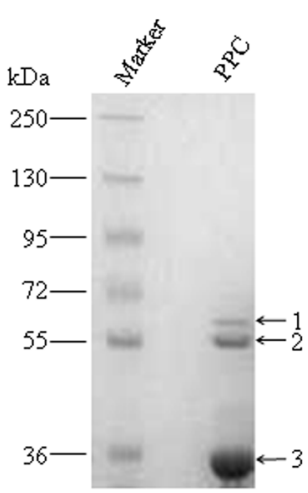

B

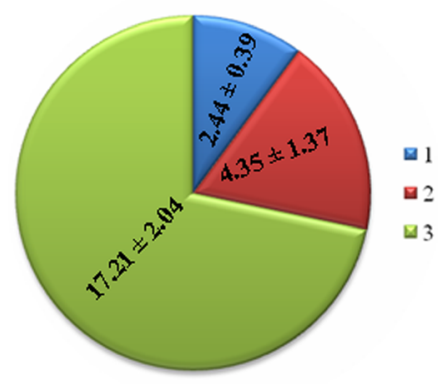

C

\begin{tabular}{|c|l|c|c|c|c|c|}
\hline $\begin{array}{c}\text { Band } \\
\text { No }\end{array}$ & \multicolumn{1}{|c|}{ Protein Name } & $\begin{array}{c}\text { NCBI accession } \\
\text { number }\end{array}$ & $\begin{array}{c}\text { Mascot } \\
\text { score }\end{array}$ & $\begin{array}{c}\text { Sequence } \\
\text { coverage } \\
(\%)\end{array}$ & $\begin{array}{c}\text { Number of } \\
\text { matched } \\
\text { peptide }\end{array}$ & $\begin{array}{c}\text { FDR } \\
\text { Values } \\
\text { (\%) }\end{array}$ \\
\hline 1 & $\begin{array}{l}\text { Pyruvate kinase, isozyme } \\
\text { M2 (EC 2.7.1.40) }\end{array}$ & KPYM_MOUSE & 185 & 21 & 11 & 0.00 \\
\hline 2 & $\begin{array}{l}\text { Glucose-6-phosphate } \\
\text { isomerase (EC 5.3.1.9) }\end{array}$ & G6PI_MOUSE & 531 & 45 & 20 & 0.00 \\
\hline 3 & $\begin{array}{l}\text { Glyceraldehyde-3- } \\
\text { phosphate dehydrogenase } \\
\text { (EC 1.2.1.12) }\end{array}$ & DEMSG & 273 & 24 & 6 & 0.00 \\
\hline
\end{tabular}

$\mathrm{D}$

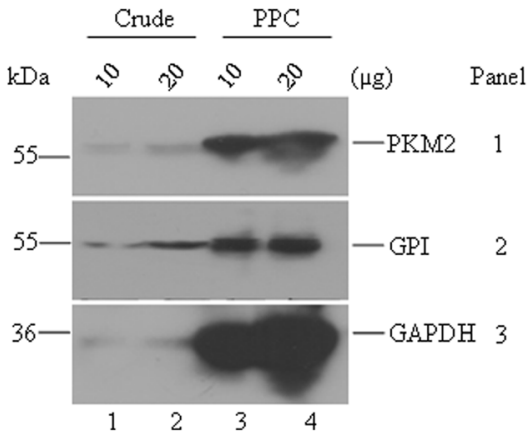

Fig. 1 GAPDH is associated with PKM2 and GPI. a A representative of coomassie blue stained 7.5-15\% Tris-glycine SDS-PAGE gel of $10 \mu \mathrm{g}$ purified protein complex (PPC) from EAC cells. Three major bands with different molecular weight approximately 58, 55, $33 \mathrm{kDa}$ are labeled with 1, 2, and 3, respectively. $\mathbf{b}$ Mole of a subunit in the complex was calculated using a formula $=[$ (intensity of the band/total intensity of three bands) $x$ loading amount\}/molecular mass]. Molar ratio is shown in pie chart. c Three bands were cut out from the gel for trypsin digestion followed by MALDI-TOF/TOF analyses. Score of identified proteins from each band is tabulated. Note that bands 1, 2 and 3 contain mainly PKM2, GPI and GAPDH respectively. Data from one representative experiment is shown here. Experiment was repeated six times. $\mathbf{d}$ Immunoblots of two different amounts of crude extract and purified protein complex (PPC) from EAC cells with antibodies specific for PKM2 (panel 1), GPI (panel 2) and GAPDH (panel 3). Note that both PKM2 and GPI were co-purified with GAPDH

(panel 3), but not GPI (panel 2), is detectable in the immunoprecipitate of PKM2, suggesting that PKM2 interacts with GAPDH, but not with GPI. Furthermore, PKM2 was undetectable in the immunoprecipitate of GPI antibody in the 3MC induced tumor tissue lysate (Fig. 3c, panel 1, lane 2), confirming that there was no interaction between GPI and PKM2 in tumor cells. Mouse or rabbit IgG was used as control antibody for immunoprecipitation. Immunoblot with secondary antibody against mouse or rabbit IgG but not primary antibody was used as a loading control for immunoprecipitate samples.
Altogether these data suggest that tumor cells show increased expression of PKM2 and GPI, and two types of GAPDH association-one is GAPDH-GPI and another is GAPDH-PKM2-exist in the 3MC induced tumor tissue.

\section{C-terminal domain of GAPDH interacts with PKM2 and GPI}

In the preceding section, we have shown that GAPDH can interact with GPI and PKM2 in cancer cells. In order to assess which domain (s) of GAPDH, PKM2 and GPI are involved in interaction; we carried out in silico molecular docking analysis. 3D structure of human 


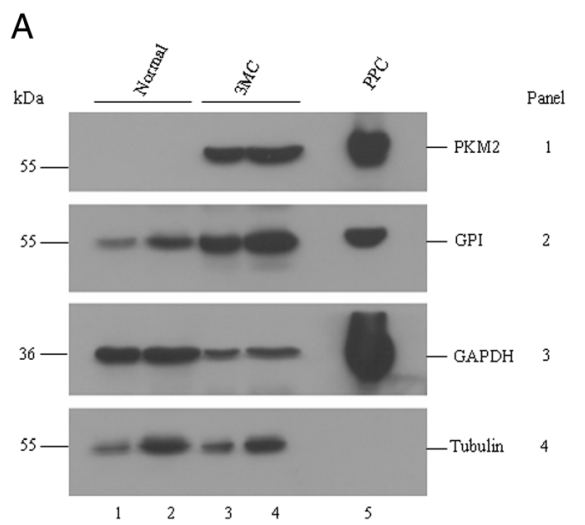

B

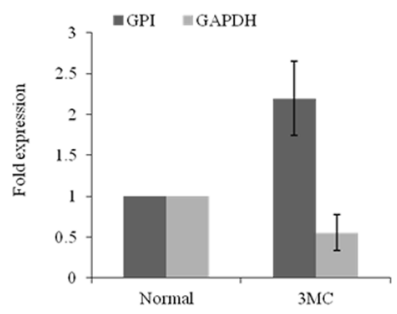

Fig. 2 Expression profile of three enzymes in mouse normal and 3MC induced tumor tissues. a Lysates were subjected to immunoblot analysis using anti-PKM2 (panel 1), -GPI (panel 2), -GAPDH (panel 3) and $\beta$-tubulin (panel 4) antibodies. $\beta$-tubulin was used as loading control for comparison of GAPDH, PKM2 or GPI expression between normal (lane 1 and 2) and tumor (lane 3 and 4) tissues. Note that the expression level of PKM2 and GPI is increased in tumor tissue. Purified protein complex (PPC) from EAC cells was considered as positive control for GAPDH, GPI, and PKM2 antibodies (lane 5). b Quantification of band intensity of the immunoblot containing GPI and GAPDH. Fold induction in each case was calculated considering the value relative band intensity for normal as "1". Results are expressed as means \pm SD from three independent experiments. ** $p<0.01$ for GPI or PKM2 in tumor vs normal

GAPDH (PDB code: 1U8F, chain O) was docked onto PKM2 (PDB code: 1ZJH, chain A) and GPI (PDB code: $1 J \mathrm{LH}$, chain A) independently without providing any prior information to the docking programs. Top docking solutions from each programs ClusPro [28, 29], PatchDock [30] and SwarmDock [31] were screened and pooled together for interface analysis. Figure 4 and Additional file 1: Figure S4 plot the overall and average frequencies of $\mathrm{N}$ or $\mathrm{C}$ terminal domain/residue involvement of GAPDH, PKM2 and GPI proteins within the GAPDHPKM2 (Fig. 4 and Additional file 1: Figure S4A-C) and
GAPDH-GPI (Fig. 4 and Additional file 1: Figure S4D-F) docking complexes, respectively. Frequencies of $\mathrm{C}$ terminal domain of GAPDH are significantly higher in GAPDHPKM2 (Fig. 4b) and GAPDH-GPI (Fig. 4e) docking complexes, advocating the role of $\mathrm{C}$ terminal part of GAPDH in interaction with both PKM2 and GPI. Similarly, $C$ terminal domain of GPI (Fig. 4f) is more likely to be used in interaction with GAPDH. However, in case of PKM2, it is not quite evident which domain is more preferred to interact with GAPDH despite of the slightly higher abundance of $\mathrm{C}$ terminal domain at the interface (Fig. 4c).

A

B

C
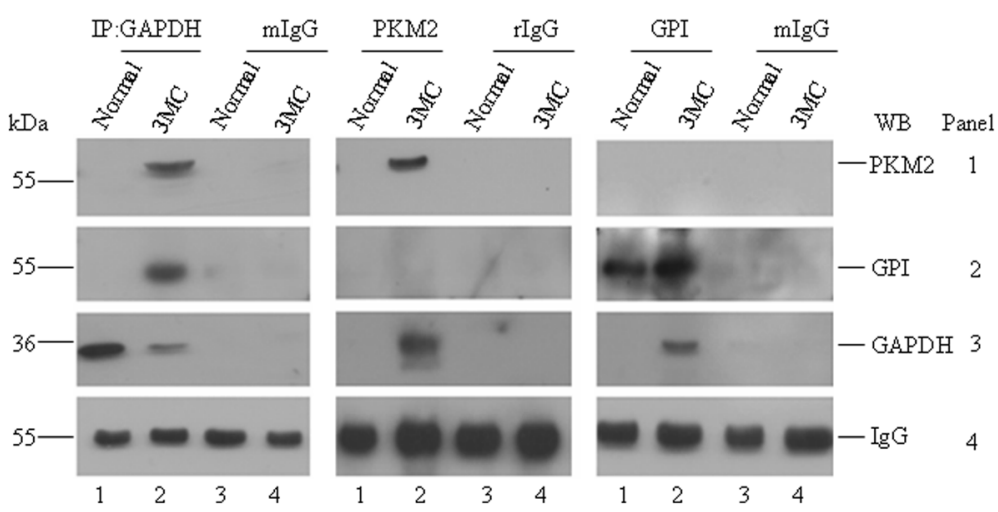

Fig. 3 Both PKM2 and GPI interact with GAPDH. a Immunoprecipitate of GAPDH antibody was subjected to immunoblot with PKM2 (panel 1), GPI (panel 2), GAPDH (panel 3) or mouse IgG heavy chain (panel 4) specific antibody. b Immunoprecipitate of PKM2 was subjected to immunoblot with PKM2, GPI, GAPDH or rabbit lgG heavy chain specific antibody. c Immunoprecipitate of GPI was subjected to immunoblot with PKM2, GPI, GAPDH or mouse lgG heavy chain specific antibody. Note that PKM2 was not immunoprecipitated with GPI antibody and vice versa. Mouse lgG was used as negative control for immunoprecipitation of GAPDH and GPI antibodies, and rabbit lgG for PKM2 (lanes 3 and 4). Antibodies against heavy chain of mouse lgG and rabbit lgG were used as loading controls for immunoblots (panel 4) 


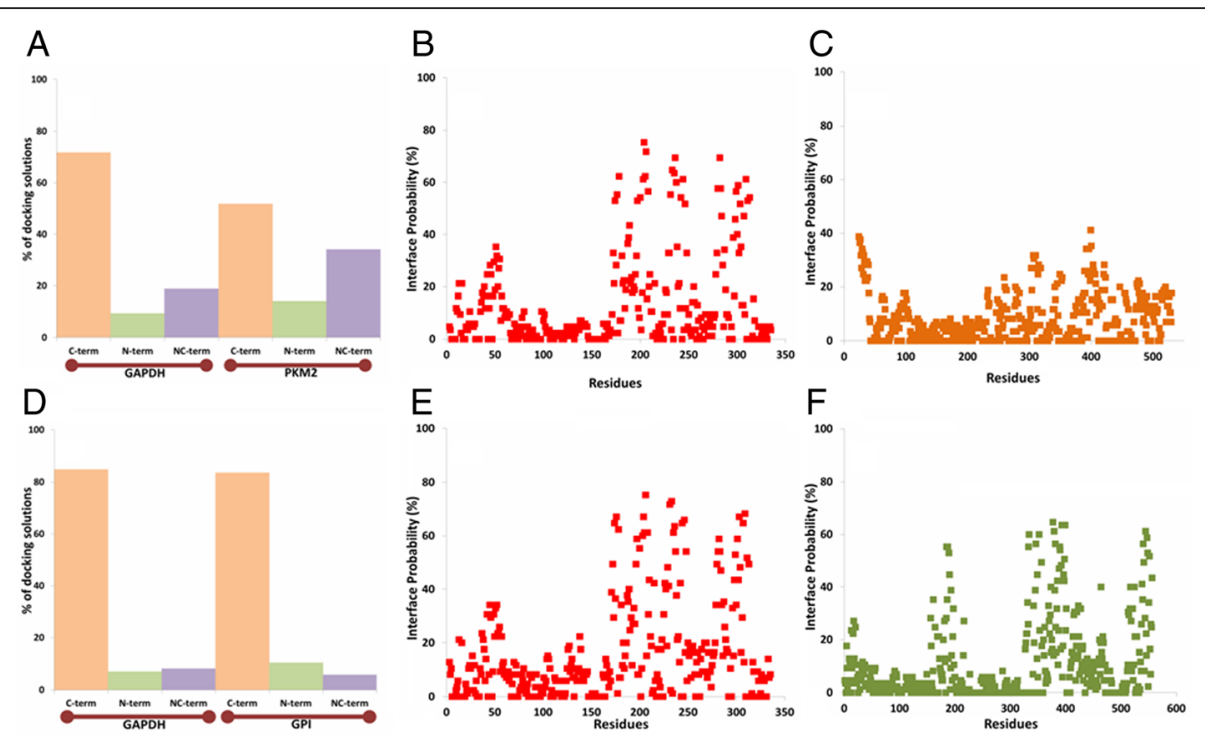

Fig. 4 Panel a provides the overall occurrence frequencies of $\mathrm{C}$ terminal, $\mathrm{N}$ terminal, and both NC of GAPDH and PKM2 proteins at the interface observed within the 90 top scoring docking complexes obtained from three different programs whereas panels $\mathbf{b}$ (GAPDH) and $\mathbf{c}$ (PKM2) provides the occurrence frequency of each residue within the GAPDH-PKM2 docking complexes. Panels $\mathbf{d}$-f represent similar domain and residue occurrence frequencies observed within the GAPDH-GPI docking complexes. An interface is regarded as C or N terminal interface if $60 \%$ of the interface residues for each protein reside within $\mathrm{C}$ or $\mathrm{N}$ termini, respectively

\section{Methylglyoxal can inhibit GAPDH activity in both EAC and 3MC induced tumor}

Ray et al. [17] showed that MG inhibits the activity of GAPDH of malignant cells but not of normal cells and benign tumor cells. We found that GAPDH is associated with two other glycolytic enzymes: PKM2 and GPI. Whether PKM2 or GPI activity is affected by MG in tumor cells, we measured the enzymatic activity of all three enzymes in EAC and 3MC induced tumor tissue in the presence of MG. Figure 5 shows that GPI activity is less likely to be inhibited by $1 \mathrm{mM} \mathrm{MG}$ in $3 \mathrm{MC}$ induced tumor (Fig. 5a) and EAC (Fig. 5b). In contrast, GAPDH activity was reduced to $26.8 \pm 5 \%$ in the $3 \mathrm{MC}$ induced tumor tissue (Fig. 5a) and to $57.8 \pm 2 \%$ in EAC cell lysates (Fig. 5b) of total activity in the presence of $1 \mathrm{mM}$ MG. We could not measure the effect of MG on the activity of pyruvate kinase (PK) in tumor cells, which may be explained due to very low activity of PK in tumor cells [33]. Additional file 1: Figure S5A shows that PK has $0.41 \pm 0.12 \mathrm{U} / \mathrm{mg}$ and $1.55 \pm 0.46 \mathrm{U} / \mathrm{mg}$ specific activity in $3 \mathrm{MC}$ and EAC, respectively, compared with $12.2 \pm 2.4 \mathrm{U} / \mathrm{mg}$ in normal tissue. We checked whether MG has any influence on the interaction of these subunits present in $3 \mathrm{MC}$ induced tumor in mouse. Additional file 1: Figure S5B shows that both PKM2 and GPI are detectable in the immunoprecipitate of GAPDH antibody
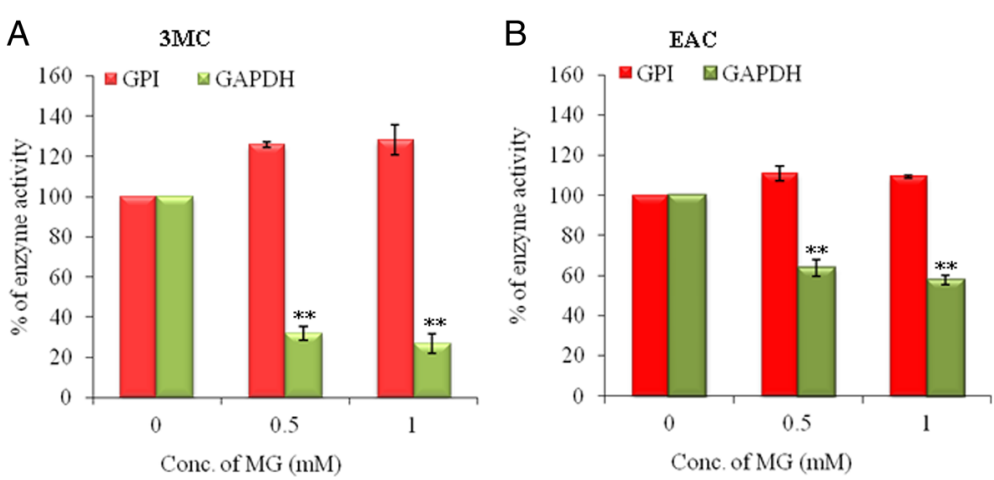

Fig. 5 MG inhibits GAPDH activity. Enzymatic activity of GPI and GAPDH in the extracts of 3MC induced tumor tissues (a) and EAC cells (b). Activity of both enzymes was measured in the absence and presence of 0.5 and $1 \mathrm{mM} \mathrm{MG}$ at $25^{\circ} \mathrm{C}$ for 10 mins. Percent inhibition was calculated considering the activity of enzyme in absence of MG as $100 \%$ (vehicle). Data presented as means \pm SD from three independent experiments. ${ }^{* *} p<0.01$ vehicle vs $0.5 \mathrm{mM}$ or $1 \mathrm{mM} \mathrm{MG}$ 
in the presence of $1 \mathrm{mM}$ MG (lane 2). Whether the inactivation by MG is due to conformational change of GAPDH complex, we carried out circular dichroism (CD) spectroscopy in the UV range with purified protein complex from EAC cells. CD spectra of untreated GAPDH complexes show two well minima at 208 and $222 \mathrm{~nm}$, which are characteristic of proteins containing $\alpha$-helical secondary structures. When these GAPDH complexes were treated with MG the minima values at these two wave lengths decreased in a dose dependent manner (Additional file 1: Figure $\mathrm{S} 5 \mathrm{C}$ ). We quantified the change of helicity in the presence of MG, and Additional file 1: Figure S5D shows that purified GAPDH complex exhibits $19 \%$ and $20 \%$ change in the helicity in the presence of $1 \mathrm{mM}$ MG for $6 \mathrm{~h}$ at 208 and $222 \mathrm{~nm}$, respectively. Taken together, these data suggest that MG may inhibit GAPDH activity by changing the helicity of GAPDH complexes (with PKM2 or GPI) without interfering the interaction with them.

\section{Methylglyoxal glycates M2 insert of PKM2 enzyme}

It is known that MG can glycate arginine and lysine residues in proteins and produce methylglyoxal advanced glycation end-products (MAGE) [19]. Using mass spectrometry analysis we checked if incubation by MG results in any glycation on arginine and lysine residues of GAPDH complex. After incubation with $1 \mathrm{mM} \mathrm{MG}$, bands correspond to PKM2, GPI and GAPDH were analyzed. Due to the mass increase characteristic of a MAGE, glycated peptides had no match in the databases and were therefore rejected [19]. This information can be used to identify the molecular location of specific MAGE in target protein(s). In the mass spectrometry of MG treated proteins, several new peaks appear that do not have predicted $\mathrm{m} / \mathrm{z}$ values. These new peaks may be due to the miscleavage of peptide(s). To know the mass of probable peptides due to miscleavage, we performed a theoretical digestion with the known protein sequence, considering upto four trypsin miscleavages, cystine alkylation with iodoacetamide and mono-isotopic peptide mass using Expasy website [19]. With these theoretical peptide masses, the mass increment due to a specific MAGE modification (54, 80 and $144 \mathrm{Da}$ for arginine, $72 \mathrm{Da}$ for lysine) were added to find out if there were any new peaks which were matched with the peak of mass spectrometry of MG treated proteins. Using this approach, no such modified peptide peaks were found in case of mass spectrometry of MG treated GAPDH (except an unknown peak at $m / z$ 2700.2021, labeled with an arrow) or GPI compared with untreated one (Additional file 1: Figure S6 A-B). Surprisingly, when the mass spectrometry peaks of PKM2 were analyzed (Fig. 6), we found that one arginine residue was modified by MG in the form of hydroimidazolone (MG-H1) (red arrow, observed mass $2141.9580 \mathrm{Da}$, corresponding to PKM2 peptide 384-400 with $\mathrm{m} / z 2087.9597$ plus $54 \mathrm{Da}$ of a MG-H modification). Detail glycated peptide sequence which was obtained by comparing with the theoretical data is shown in Additional file 1: Table S4. Glycated peptide has miscleavage in $\mathrm{Arg}^{399}$ residue. In contrast, no such peak with $\mathrm{m} / \mathrm{z}$ value of $2141.9580 \mathrm{Da}$ was found in the untreated mass spectrometry. These data suggest that MG can glycate Arg ${ }^{399}$ in PKM2 of purified GAPDH complex.

Previous report [34] revealed that the positively charged residue R399 of PKM2 may play a critical role in forming the PKM2 tetramer by a stable charge-charge interactions with residues E418 and E396 of PKM2 which are located on the other dimer of the tetramer PKM2. Replacing Arginine with Glutamic acid disrupts such interaction and prefers dimmer, not tetramer. When R399 was mutated with Alaline, mutant PKM2 was unable to translocate to the nucleus in EGF mediated translocation, and unable to interact with importin which facilitate nuclear translocation [35]. We assessed biochemical function of R399 residue of PKM2 using in silico molecular docking analysis at the interface with GAPDH. Figure 7a shows higher abundance of Arginine 399 (R399) and its neighboring residues (within $5 \AA$ ) at the interface with GAPDH. Higher frequency of hydrogen bonding (Fig. 7b) and salt bridge formation (Fig. 7c), while lower value of average $\Delta \mathrm{G}$ of interaction (Fig. $7 \mathrm{~d}$ ) at the interface suggest the importance of R399 and its neighboring residues in the interaction with GAPDH.

\section{Discussion}

In this paper we demonstrate that association of GAPDH with GPI or PKM2 exists in both EAC cells and 3MC induced tumor tissue. MG can glycate PKM2 at R399 residue, which may induce structural changes in GAPDH complex.

GAPDH was considered as a stable housekeeping marker for constant gene expression [36]. However, recent times have seen the ongoing discovery of new roles for GAPDH in a diverse range of cellular processes which clearly demonstrate that there are more complex roles of GAPDH in case of cellular metabolism [37, 38]. Li et al. [39] reports that GAPDH is physically associated with p300/CREB binding protein associated factor (PCAF), and histone deacetylase 5 (HDAC5) for its higher enzymatic activity by acetylation at active site's lysine 254 residue in HEK 293 T and A549 lung cancer cell line. GAPDH binds with SET protein to regulate cyclin B-cdk1 activity [40]. However we could not detect any association of these proteins with GAPDH in the cells that we have used in our study. This may be due to a different mechanism associated with post translational modification based on regulation in glycolysis pathway in different cancer cells. Interestingly, we could detect new peaks in the MG treated sample of GAPDH, GPI and PKM2 that do not have predicted $\mathrm{m} / \mathrm{z}$ 


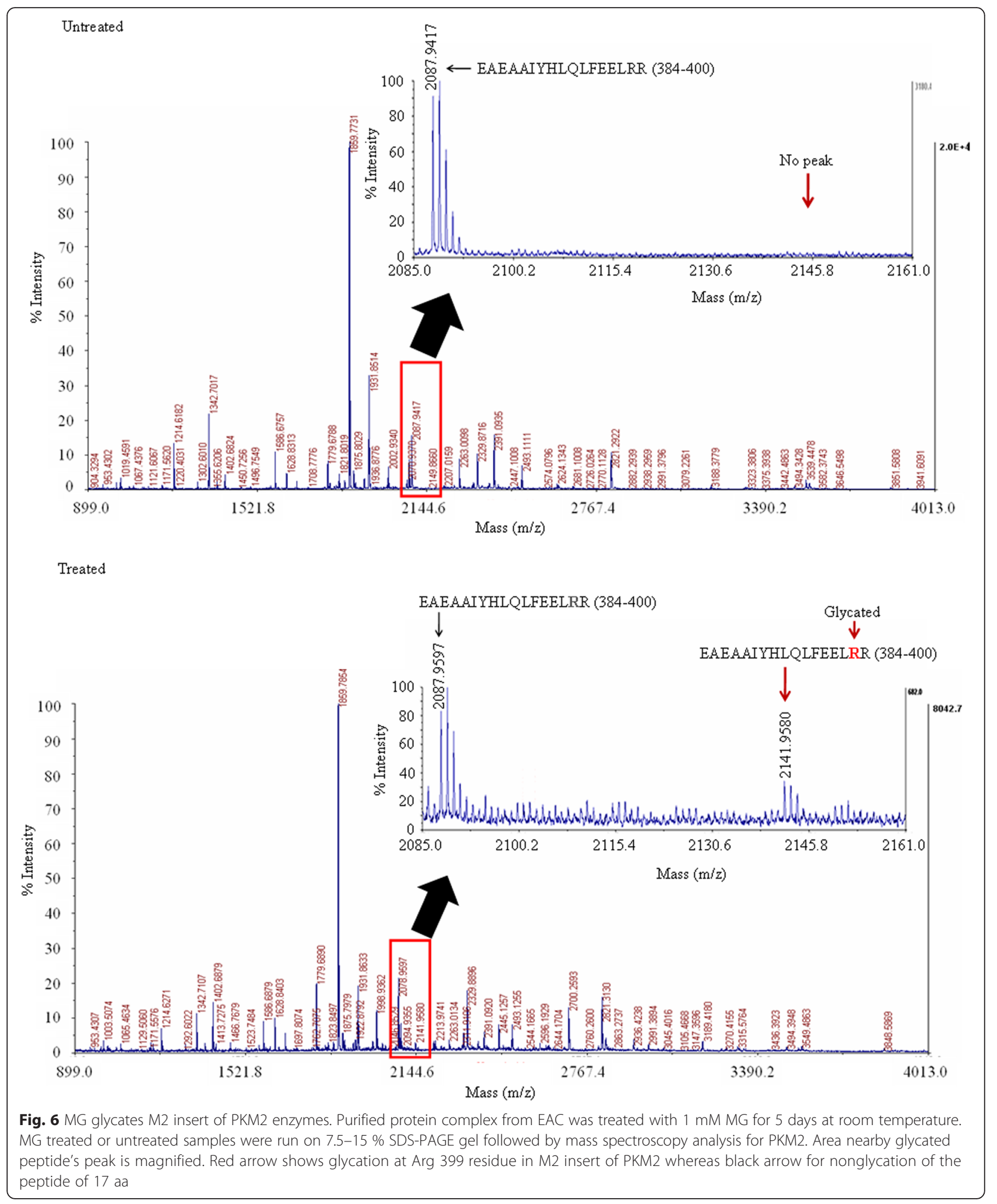

values. One major peak was observed in case of treated GAPDH at $m / z$ value 2700 compared with untreated one (Additional file 1: Figure S6A). But, the type of modification is still unknown to us because this peak value does not match with any theoretical peptide value after addition of the mass increment due to MAGE modification. We can not rule out the possibility of such modification in GAPDH by MG, which may inhibit its enzymatic activity 


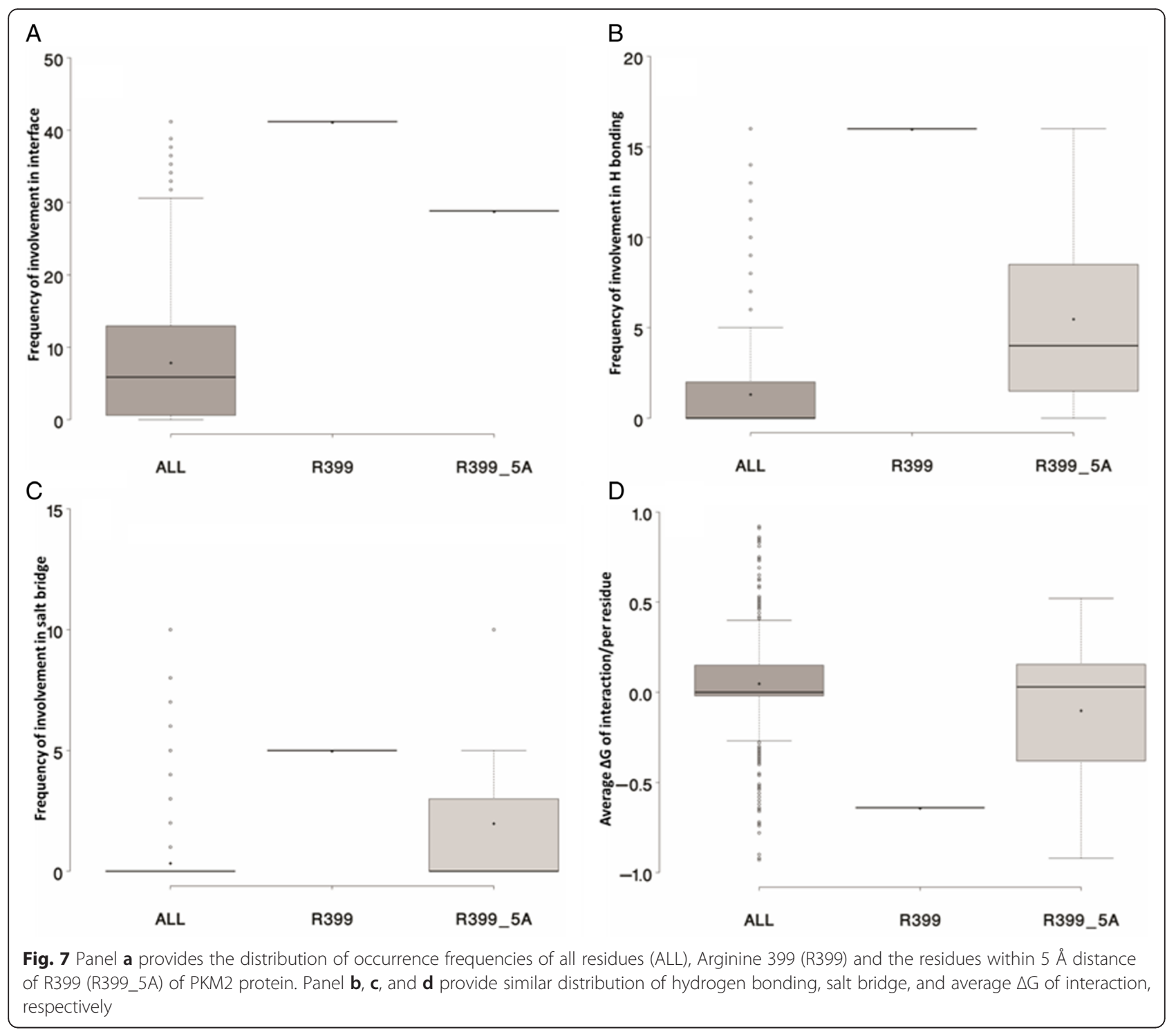

in cancer cells. Further studies are being carrying out in the laboratory to decipher the kind of modification occurred in the presence of MG.

Pyruvate kinase of muscle cells (PKM) exist in two isoforms: PKM1 and PKM2. They are generated due to alternative splicing [41]. PKM2 regulates many metabolic pathways for cancer cell survival and proliferation [34, 42-45]. PKM2 activity supports Warburg effect for cancer cell survival $[35,46]$. We checked the expression profile of PKM isoforms in normal and $3 \mathrm{MC}$ induced-tumor tissues. Interestingly, $\mathrm{PKM} 2$ was detectable in $3 \mathrm{MC}$ induced tumor whereas PKM1 in normal tissue (Fig. 2a and Additional file 1: Figure S2). MG could glycate $\mathrm{Arg}^{399}$, which is located in alternatively spliced M2 insert of PKM2 of purified GAPDH complex. These observations led us to hypothesize that alternative splicing of PKM gene is an important factor for association of GAPDH with PKM2, which could be one of the ways for cancer cells to increase high glycolytic activity. Glycation at $\mathrm{Arg}^{399}$ of PKM2 followed by conformational changes of the GAPDH complexes could be one of the possible mechanisms for MG mediated inhibition of glycolysis in tumor cells.

Recently, glycolytic enzyme, GPI has been shown to regulate tumor cell growth and metastasis. Down regulation of GPI increases sensitivity towards oxidative stress and oxidative stress induced senescence [47]. Funasaka et al. [48] have shown that hypoxia increases GPI expression in human breast carcinoma BT-549 cells. Inhibition of GPI expression by siRNA reduces hypoxia induced cancer cell motility. Association of GAPDH with PKM2 and GPI may give a special advantage for cancer cell metabolism and survival. This association could be a signature for cancer cells. Further studies are needed to understand how interaction of GPI and PKM2 alters the activity of 
GAPDH, and helps cancer cell metabolism for developing a therapeutic approach for treatment of cancers.

\section{Conclusion}

GAPDH interacts with an alternatively spliced isoforms of pyruvate kinase, PKM2 and GPI in cancer cells. These interactions may be one of the reasons for cancer cells to exhibit high glycolytic activity. MG can glycate at R399 residue, located in M2 insert, of PKM2 and inhibits the GAPDH activity. This could be one of the reasons for MG mediated inhibition of glycolysis in cancer cells.

\section{Additional file}

\section{Additional file 1: Supplementary material. (PDF $623 \mathrm{~kb}$ )}

\begin{abstract}
Abbreviations
GAP: Glyceraldehyde-3-phosphate; GAPDH: Glyceraldehyde-3-phosphate dehydrogenase; GPI: Glucose-6-phosphate isomerase; IP: Immunoprecipitation; MAGE: Methylglyoxal-derived advanced glycation end-products; MALDI-TOF: Matrix-assisted laser desorption ionization-time of flight; 3MC: 3-Methylcholanthrene; MG: Methylglyoxal; PKM: Pyruvate kinase muscle; PKM1: Pyruvate kinase M1; PKM2: Pyruvate kinase M2; RIPA: Radioimmunoprecipitation assay.
\end{abstract}

\section{Competing interests}

The authors declare that they have no competing interests.

\section{Authors' contributions}

MRD designed and conducted all studies with the help of other co-authors- AKB, SS, AG, SKD, and PD. AKB, MRD, SSJ and CM analyzed the mass spectrometry data, and SC carried out in silico molecular docking analysis. SSJ and MR conceived the study, discussed in analyzing the data. MRD, SSJ, SR, and SC wrote the manuscript. All the authors have read and approved the final manuscript.

\section{Acknowledgements}

This work is dedicated to the fond memory of Dr. Subhankar Ray. We thank Debdatta Halder for critically reading the manuscript. We also thank Drs. Parthasarathi Dastidar and Jolly Deb for reagents and helpful discussion. We thank Indian Association for the Cultivation of Science, Indian Institute of Chemical Biology, Department of Biotechnology, Department of Science and Technology, Government of India, J. C Bose National Fellowship to Dr. C Mandal, Council of Scientific and Industrial Research fellowship to PD and SKD Indian Council of Medical Research fellowship to AKB for financial assistance.

\section{Author details}

${ }^{1}$ Department of Biological Chemistry, Indian Association for the Cultivation of Science, Kolkata 700032, India. ${ }^{2}$ Cancer Biology and Inflammatory Disorder Division, Indian Institute of Chemical Biology, Kolkata 700032, India. ${ }^{3}$ Department of Biochemistry and Biophysics, Texas A\&M University, College Station, TX 77843-3474, USA. ${ }^{4} 13$ Regent Estate, Kolkata 700 092, India. ${ }^{5}$ Structural Biology and Bioinformatics Division, Indian Institute of Chemical Biology, Jadavpur, Kolkata 700 032, India. ${ }^{6}$ Department of Biophysics, Bose Institute, Kolkata, India.

Received: 2 April 2015 Accepted: 14 February 2016 Published online: 24 February 2016

\section{References}

1. Warburg O. On the origin of cancer cells. Science. 1956;123:309-14.

2. Saunders PA, Chen RW, Chuang DM. Nuclear translocation of glyceraldehyde-3phosphate dehydrogenase isoforms during neuronal apoptosis. J Neurochem. 1999;72:925-32.
3. Laschet JJ, Minier F, Kurcewicz I, Bureau MH, Trottier S, Jeanneteau F, et al Glyceraldehyde-3-phosphate dehydrogenase is a GABAA receptor kinase linking glycolysis to neuronal inhibition. J Neurosci. 2004;24:7614-22.

4. Ravichandran V, Seres T, Moriguchi T, Thomas JA, Johnston Jr RB. Sthiolation of glyceraldehyde-3-phosphate dehydrogenase induced by the phagocytosis-associated respiratory burst in blood monocytes. J Biol Chem. 1994;269:25010-5.

5. Glaser PE, Han X, Gross RW. Tubulin is the endogenous inhibitor of the glyceraldehyde 3-phosphate dehydrogenase isoform that catalyzes membrane fusion: implications for the coordinated regulation of glycolysis and membrane fusion. Proc Natl Acad Sci U S A. 2002:99:14104-9.

6. Tisdale E. Glyceraldehyde-3-phosphate dehydrogenase is required for vesicular transport in the early secretory pathway. J Biol Chem. 2001;276:2480-6.

7. Nakajima H, Amano W, Kubo T, Fukuhara A, Ihara H, Azuma YT, et al. Glyceraldehyde-3-phosphate dehydrogenase aggregate formation participates in oxidative stress-induced cell death. J Biol Chem. 2009;284:34331-41.

8. You B, Huang S, Qin Q, Yi B, Yuan Y, Xu Z, et al. Glyceraldehyde-3-phosphate dehydrogenase interacts with proapoptotic kinase Mst1 to promote cardiomyocyte apoptosis. PLoS One. 2013:8:e58697.

9. Huang Q, Lan F, Zheng Z, Xie F, Han J, Dong L, et al. Akt2 kinase suppresses glyceraldehyde-3-phosphate dehydrogenase (GAPDH)-mediated apoptosis in ovarian cancer cells via phosphorylating GAPDH at threonine 237 and decreasing its nuclear translocation. J Biol Chem. 2011;286:42211-20.

10. Tokunaga K, Nakamura Y, Sakata K, Fujimori K, Ohkubo M, Sawada K, et al. Enhanced expression of a glyceraldehyde-3-phosphate dehydrogenase gene in human lung cancers. Cancer Res. 1987;47:5616-9.

11. Schek N, Hall BL, Finn OJ. Increased glyceraldehyde-3-phosphate dehydrogenase gene expression in human pancreatic adenocarcinoma. Cancer Res. 1988;48:6354-9.

12. Epner DE, Partin AW, Schalken JA, Isaacs JT, Coffey DS. Association of glyceraldehyde-3-phosphate dehydrogenase expression with cell motility and metastatic potential of rat prostatic adenocarcinoma. Cancer Res. 1993;53:1995-7

13. Wang D, Moothart DR, Lowy DR, Qian X. The expression of glyceraldehyde3-phosphate dehydrogenase associated cell cycle (GACC) genes correlates with cancer stage and poor survival in patients with solid tumors. PLoS One. 2013;8:e61262.

14. Ferreira-da-Silva F, Pereira PJ, Gales L, Roessle M, Svergun DI, Moradas-Ferreira P, et al. The crystal and solution structures of glyceraldehyde-3-phosphate dehydrogenase reveal different quaternary structures. J Biol Chem. 2006;281:33433-40

15. Bagui S, Ray M, Ray S. Glyceraldehyde-3-phosphate dehydrogenase from Ehrlich ascites carcinoma cells. Its possible role in the high glycolysis of malignant cells. Eur J Biochem. 1999;262:386-95

16. Patra S, Ghosh S, Bera S, Roy A, Ray S, Ray M. Molecular characterization of tumor associated glyceraldehyde-3-phosphate dehydrogenase. Biochemistry. 2009;74:717-27.

17. Ray M, Basu N, Ray S. Inactivation of glyceraldehyde-3-phosphate dehydrogenase of human malignant cells by methylglyoxal. Mol Cell Biochem. 1997;177:21-6.

18. Ray S, Dutta S, Halder J, Ray M. Inhibition of electron flow through complex I of the mitochondrial respiratory chain of Ehrlich ascites carcinoma cells by methylglyoxal. Biochemical J. 1994;303:69-72.

19. Gomes RA, Vicente MH, Silva MS, Graça G, Coelho AV, Ferreira AE, et al. Yeast protein glycation in vivo by methylglyoxal. Molecular modification of glycolytic enzymes and heat shock proteins. FEBS J. 2006;273:5273-87.

20. Khatua B, Van Vleet J, Choudhury BP, Chaudhry R, Mandal C. Sialylation of outer membrane porin protein D: a mechanistic basis of antibiotic uptake in Pseudomonas aeruginosa. Mol Cell Proteomics. 2014;13:1412-28.

21. Zhang Q, Ames JM, Smith RD, Baynes JW, Metz TO. A perspective on the Maillard reaction and the analysis of protein glycation by mass spectrometry: probing the pathogenesis of chronic disease. J Proteome Res. 2009;8:754-69.

22. Saha S, Dey SK, Das P, Jana SS. Increased expression of nonmuscle myosin Ils is associated with 3MC-induced mouse tumor. FEBS J. 2011;278:4025-34.

23. Saha S, Dey SK, Biswas A, Das P, Das MR, Jana SS. The effect of including the $\mathrm{C} 2$ insert of nonmuscle myosin II-C on neuritogenesis. J Biol Chem. 2013:288:7815-28

24. Roe $\mathrm{JH}$. A colorimetric method for the determination of fructose in blood and urine. J Biol Chem. 1934:1934(107):15-22.

25. Jenkins JL, Tanner JJ. High-resolution structure of human D-glyceraldehyde3-phosphate dehydrogenase. Acta Crystallogr D Biol Crystallogr. 2006;62:290-301 
26. Cordeiro AT, Godoi PH, Silva CH, Garratt RC, Oliva G, Thiemann OH. Crystal structure of human phosphoglucoseisomerase and analysis of the initial catalytic steps. Biochim Biophys Acta. 2003;1645:117-22.

27. Berman HM, Westbrook J, Feng Z, Gilliland G, Bhat TN, Weissig H, et al. The protein data bank. Nucleic Acids Res. 2000;28:235-42.

28. Kozakov D, Beglov D, Bohnuud T, Mottarella S, Xia B, Hall DR, et al. How good is automated protein docking? Proteins. 2013;81:2159-66.

29. Kozakov D, Brenke R, Comeau SR, Vajda S. PIPER: An FFT-based protein docking program with pairwise potentials. Proteins. 2006;65:392-406.

30. Schneidman-Duhovny D, Inbar Y, Nussinov R, Wolfson HJ. PatchDock and SymmDock: Servers for rigid and symmetric docking. Nucleic Acids Res. 2005;33(Web Server issue):W363-367.

31. Torchala M, Moal IH, Chaleil RAG, Fernandez-Recio J, Bates PA. SwarmDock: A server for flexible protein-protein docking. Bioinformatics. 2013;29:807-9.

32. Krissinel $\mathrm{E}$, Henrick K. Inference of macromolecular assemblies from crystalline state. J Mol Biol. 2007;372:774-97.

33. Christofk HR, Vander HMG, Harris MH, Ramanathan A, Gerszten RE, Wei R, et al. The M2 splice isoform of pyruvate kinase is important for cancer metabolism and tumour growth. Nature. 2008;452:230-3.

34. Gao X, Wang H, Yang JJ, Liu X, Liu ZR. Pyruvate kinase M2 regulates gene transcription by acting as a protein kinase. Mol Cell. 2012;45:598-609.

35. Yang W, Zheng Y, Xia Y, Ji H, Chen X, Guo F, et al. ERK1/2-dependent phosphorylation and nuclear translocation of PKM2 promotes the Warburg effect. Nat Cell Biol. 2012;14:1295-304.

36. Ferguson RE, Carroll HP, Harris A, Maher ER, Selby PJ, Banks RE. Housekeeping proteins: a preliminary study illustrating some limitations as useful references in protein expression studies. Proteomics. 2005;5:566-71.

37. Tristan C, Shahani N, Sedlak TW, Sawa A. The diverse functions of GAPDH: Views from different subcellular compartments. Cell Signal. 2011;23:317-23.

38. Sawa A, Khan AA, Hester LD, Snyder SH. Glyceraldehyde-3-phosphate dehydrogenase: nuclear translocation participates in neuronal and nonneuronal cell death. Proc Natl Acad Sci USA. 1997:94:11669-74.

39. Li T, Liu M, Feng X, Wang Z, Das I, Xu Y, et al. Glyceraldehyde-3-phosphate dehydrogenase is activated by lysine 254 acetylation in response to glucose signal. J Biol Chem. 2014;289:3775-85.

40. Carujo S, Estanyol JM, Ejarque A, Agell N, Bachs O, Pujol MJ. Glyceraldehyde 3-phosphate dehydrogenase is a SET-binding protein and regulates cyclin B-cdk1 activity. Oncogene. 2006;25:4033-42.

41. Noguchi T, Inoue H, Tanaka T. The M1- and M2-type isozymes of rat pyruvate kinase are produced from the same gene by alternative RNA splicing. J Biol Chem. 1986;261:13807-12.

42. Anastasiou D, Yu Y, Israelsen WJ, Jiang JK, Boxer MB, Hong BS, et al. Pyruvate kinase M2 activators promote tetramer formation and suppress tumorigenesis. Nat Chem Biol. 2012;8:839-47.

43. Cortés-Cros M, Hemmerlin C, Ferretti S, Zhang J, Gounarides JS, Yin H, et al. M2 isoform of pyruvate kinase is dispensable for tumor maintenance and growth. Proc Natl Acad Sci U S A. 2013;110:489-94.

44. Luo W, Hu H, Chang R, Zhong J, Knabel M, O'Meally R, et al. Pyruvate Kinase M2 is a PHD3-stimulated coactivator for hypoxia-inducible factor 1. Cell. 2011:145:732-44.

45. Mazurek S, Grimm H, Boschek CB, Vaupel P, Eigenbrodt E. Pyruvate kinase type M2: a crossroad in the tumor metabolome. Br J Nutr. 2002;87 Suppl 1:S23-29.

46. Yang $W$, Xia Y, Ji H, Zheng Y, Liang J, Huang $W$, et al. Nuclear PKM2 regulates B-catenin transactivation upon EGFR activation. Nature. 2011:480:118-22.

47. Funasaka T, Hu H, Hogan V, Raz A. Down-regulation of phosphoglucose isomerase/autocrine motility factor expression sensitizes human fibrosarcoma cells to oxidative stress leading to cellular senescence. J Biol Chem. 2007;282:36362-9.

48. Funasaka T, Yanagawa T, Hogan V, Raz A. Regulation of phosphoglucose isomerase/autocrine motility factor expression by hypoxia. FASEB J. 2005:19:1422-30.

\section{Submit your next manuscript to BioMed Central and we will help you at every step:}

- We accept pre-submission inquiries

- Our selector tool helps you to find the most relevant journal

- We provide round the clock customer support

- Convenient online submission

- Thorough peer review

- Inclusion in PubMed and all major indexing services

- Maximum visibility for your research

Submit your manuscript at www.biomedcentral.com/submit
Biomed Central 